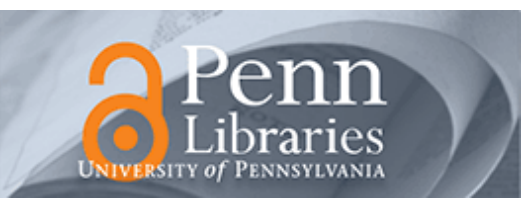

University of Pennsylvania

ScholarlyCommons

$6-2009$

\title{
Non-Rigid Registration between Histological and MR Images of the Prostate: A Joint Segmentation and Registration Framework
}

\author{
Yangming $\mathrm{Ou}$ \\ University of Pennsylvania, ouya@seas.upenn.edu \\ Dinggang Shen \\ University of Pennsylvania, Dinggang.Shen@uphs.upenn.edu \\ Michael Feldman \\ University of Pennsylvania, feldmanm@mail.med.upenn.edu \\ John Tomaszewski \\ University of Pennsylvania, TOMASZEW@MAIL.MED.UPENN.EDU \\ Christos Davatzikos \\ University of Pennsylvania, christos@rad.upenn.edu \\ Follow this and additional works at: https://repository.upenn.edu/be_papers \\ Part of the Bioimaging and Biomedical Optics Commons
}

\section{Recommended Citation}

Ou, Y., Shen, D., Feldman, M., Tomaszewski, J., \& Davatzikos, C. (2009). Non-Rigid Registration between Histological and MR Images of the Prostate: A Joint Segmentation and Registration Framework. Retrieved from https://repository.upenn.edu/be_papers/141

Yangming Ou, Dinggang Shen, Michael Feldman, John Tomaszewski, Christos Davatzikos. "Non-Rigid Registration between Histological and MR Images of the Prostate: A Joint Segmentation and Registration Framework".

Computer Vision and Pattern Recognition (CVPR) Workshop: Mathematical Methods in Biomedical Image Analysis (MMBIA), Miami, FL, 2009: pp. 125-132.

http://www.seas.upenn.edu/ ouya/documents/research/Ou09_MMBIA.pdf

This paper is posted at ScholarlyCommons. https://repository.upenn.edu/be_papers/141

For more information, please contact repository@pobox.upenn.edu. 


\title{
Non-Rigid Registration between Histological and MR Images of the Prostate: A Joint Segmentation and Registration Framework
}

\author{
Abstract \\ This paper presents a 3D non-rigid registration algorithm between histological and MR images of the \\ prostate with cancer. To compensate for the loss of 3D integrity in the histology sectioning process, \\ series of 2D histological slices are first reconstructed into a 3D histological volume. After that, the 3D \\ histology-MRI registration is obtained by maximizing a) landmark similarity and b) cancer region overlap \\ between the two images. The former aims to capture distortions at prostate boundary and internal \\ bloblike structures; and the latter aims to capture distortions specifically at cancer regions. In particular, \\ landmark similarities, the former, is maximized by an annealing process, where correspondences between \\ the automatically-detected boundary and internal landmarks are iteratively established in a fuzzy-to- \\ deterministic fashion. Cancer region overlap, the latter, is maximized in a joint cancer segmentation and \\ registration framework, where the two interleaved problems - segmentation and registration - inform \\ each other in an iterative fashion. Registration accuracy is established by comparing against human-rater- \\ defined landmarks and by comparing with other methods. The ultimate goal of this registration is to warp \\ the histologically-defined cancer ground truth into MRI, for more thoroughly understanding MRI signal \\ characteristics of the prostate cancerous tissue, which will promote the MRI-based prostate cancer \\ diagnosis in the future studies.

\section{Keywords} \\ Image Registration, Histological Image, MR Image, Deformable Registration, Non-Rigid Registration

\section{Disciplines} \\ Bioimaging and Biomedical Optics

\section{Comments} \\ Yangming Ou, Dinggang Shen, Michael Feldman, John Tomaszewski, Christos Davatzikos. "Non-Rigid \\ Registration between Histological and MR Images of the Prostate: A Joint Segmentation and Registration \\ Framework". Computer Vision and Pattern Recognition (CVPR) Workshop: Mathematical Methods in \\ Biomedical Image Analysis (MMBIA), Miami, FL, 2009: pp. 125-132. \\ http://www.seas.upenn.edu/ ouya/documents/research/Ou09_MMBIA.pdf
}




\title{
Non-Rigid Registration between Histological and MR Images of the Prostate: A Joint Segmentation and Registration Framework
}

\author{
Yangming $\mathrm{Ou}^{1, *}$, Dinggang Shen ${ }^{2}$, Michael Feldman ${ }^{3}$, John Tomaszewski ${ }^{3}$ and Christos Davatzikos ${ }^{1}$ \\ ${ }^{1}$ Dept. of Radiology, ${ }^{3}$ Dept. of Pathology, UPenn, Philadelphia, PA, 19104 \\ ${ }^{2}$ Dept. of Radiology, UNC-Chapel Hill, Chapel Hill, NC, 27510
}

\begin{abstract}
This paper presents a $3 D$ non-rigid registration algorithm between histological and MR images of the prostate with cancer. To compensate for the loss of $3 D$ integrity in the histology sectioning process, series of $2 D$ histological slices are first reconstructed into a $3 D$ histological volume. After that, the $3 D$ histology-MRI registration is obtained by maximizing a) landmark similarity and $b$ ) cancer region overlap between the two images. The former aims to capture distortions at prostate boundary and internal bloblike structures; and the latter aims to capture distortions specifically at cancer regions. In particular, landmark similarities, the former, is maximized by an annealing process, where correspondences between the automatically-detected boundary and internal landmarks are iteratively established in a fuzzy-to-deterministic fashion. Cancer region overlap, the latter, is maximized in a joint cancer segmentation and registration framework, where the two interleaved problems - segmentation and registration - inform each other in an iterative fashion. Registration accuracy is established by comparing against human-rater-defined landmarks and by comparing with other methods. The ultimate goal of this registration is to warp the histologically-defined cancer ground truth into MRI, for more thoroughly understanding MRI signal characteristics of the prostate cancerous tissue, which will promote the MRI-based prostate cancer diagnosis in the future studies.
\end{abstract}

\section{Introduction}

Over decades of technological and clinical progress, Magnetic Resonance Imaging (MRI) has emerged as one of the most important tools for diagnosing abnormalities and cancers in a variety of human organs. Recently, MRI has been increasingly used to diagnose prostate cancer, the most common cancer and the second leading cause for cancerrelated death in American men [3]. However, limited diagnostic accuracy has been reported $[1,2]$, mainly because the relationship between the MRI imaging signals and the underlying anatomic properties of the prostate cancerous tissue is far from being completely understood. To more thoroughly understand the MRI signal characteristics of the prostate cancerous tissue, cancer ground truth is required in the prostate MR images.

To label cancer ground truth in prostate MR images, one often has to refer to histological images of the same prostate. This is because histology could reveal the underlying anatomic reality of the cancerous tissue at the micro level, providing the authentic cancer ground truth. To warp the histologically-defined cancer ground truth to MR images, registration between the two images is required. Therefore, this paper focuses on registration between histological and MR images of the same prostate.

Histology-MRI registration is often severely challenged in the following two respects. The first challenge is the 2D/3D distortions in histological images. The distortions are introduced when the prostate is extracted from the body and embedded into a paraffin box, causing inevitable dehydration, tissue extraction and the loss of blood irrigation. Distortions are also introduced when the prostate is sectioned into series of 2D slices, causing the loss of 3D integrity. In the presence of those 2D/3D distortions, registration between histological and MR images has to be nonrigid, although they are acquired from the same prostate.

The second challenge is the inherent differences of the imaging characteristics between histology and MRI. As can be observed in Fig. 1, those inherent differences cause certain features and contrasts evidently visible in one image but hardly visible in the other. Meanwhile, the intensity distributions between the two images do not follow a consistent relationship, which violates the fundamental assumption of the commonly-used mutual information (MI) [4] based registration methods. Therefore, an ideal registration method should have a robust similarity metric to establish correspondences between those two images.

Because of the aforementioned challenges, the literature specifically dealing with histology-MRI registration is rel- 

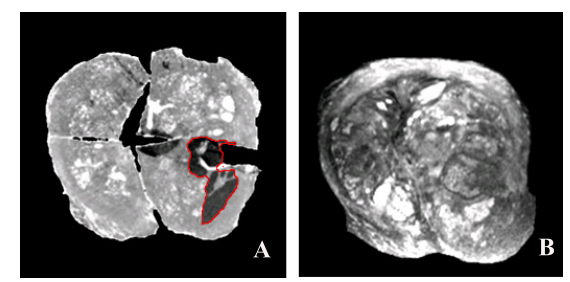

Figure 1. Typical histological image (A) and MR image (B) of the same prostate. Cancer ground truth is defined in histology (inside the red contour in subfigure (A)), but largely unknown and left to be estimated in MRI (B). For display purpose, only 2D slices are shown, but our algorithm is in 3D.

atively limited. Pioneer work [5, 6] used affine registration to align histology-MRI of the brain and presented promising results, but affine transformation has limited ability to deal with the non-linear distortions. To deal with nonlinear distortions, Pitiot et al [7] automatically partitioned images into pieces and used piece-wise affine model with the final integrated deformable deformations. They have shown impressive results in brain images, but the prostate images are more difficult to be naturally partitioned into anatomically-meaningful pieces. Another approach by Jacobs et al [8] used a surface matching method to align rat brain boundaries, followed by the thin-plate-spline based warping, but the internal distortions were not well captured. To better capture internal distortions, other methods [9][10][11] built correspondences on internal landmarks, but the landmark detection and matching processes often need human interventions, which is time-consuming and irreproducible. To avoid human interventions, recent work [12][13][14] automated the landmark detection and matching processes. In their work, the internal landmarks are automatically detected as those anatomically-salient blob-like structures. However, due to the lack of blob-like structures within and around cancer regions, few or no internal landmarks can be detected there. Consequently, the distortions at cancer regions are less likely to be captured. This causes a severe problem, especially when cancer regions are the main regions of interest in this histology-MRI registration context (keep in mind that, our goal in this paper is to warp the histologically-defined cancer regions into MR image).

This paper presents a novel 3D non-rigid registration algorithm between histological and MR images of the prostate. Our algorithm initializes with the coarse reconstruction of the series of $2 \mathrm{D}$ histological slices into a $3 \mathrm{D}$ volume, in order to recover 3D integrity of histology. Then, 3D histological and 3D MR images are registered based on two criteria: maximization of landmark similarities and maximization of cancer region overlap. The former aims to capture 2D/3D distortions in the boundary and internal (non-cancer) regions. The latter aims to capture distortions specifically within and around cancer regions, which can be hardly captured by the internal landmarks in the first crite- rion. In particular, the former is obtained by an annealing process where correspondences on boundary and internal landmarks are established in a fuzzy-to-deterministic fashion. The latter is obtained by a joint cancer segmentation and registration framework, where the two interleaved problems - segmentation and registration - benefit each other in an iterative fashion. Finally, the registration accuracy is established by comparing against human-rater-defined landmarks and by comparing with other methods.

This paper builds upon the work of [13] and has the following two extensions. First, the landmark-based direct registration method in [13] is now extended into a joint cancer segmentation and registration framework, using the registration result from [13] as the initialization. In the joint framework, the two interleaved processes - segmentation and registration - benefit each other and iteratively improve the overall accuracy, especially the accuracy at the cancer regions, which are the regions of interest in this histologyMRI context (since our objective is to use the registration to warp cancer regions from histology to MRI). Second, the forward landmark matching (histology $\rightarrow$ MRI) in [13] is extended to a forward-backward landmark matching (histology $\leftrightarrow$ MRI) in this work, therefore more reliable correspondences can be established [21], and better initialization result can be obtained for the subsequent joint cancer segmentation and registration framework.

The remainder of this paper is organized as follows. Section 2 presents details of the registration algorithm, with results on simulated and real data shown in Section 3. The whole paper is discussed and concluded in Section 4.

\section{Method}

\subsection{Data Acquisition}

T2-weighted MR images are acquired with a whole body Siemens Trio MR scanner, using fast spin echo (FSE) sequence, TE $126 \mathrm{msec}$., TR $3000 \mathrm{msec} ., 15.6 \mathrm{khz}$, and 4 signal averages. The MR image size is typically $256 \times 256 \times 64$ voxels and voxel size is $0.15 \mathrm{~mm} \times 0.15 \mathrm{~mm} \times 0.75 \mathrm{~mm}$. Histological slices are acquired in four steps: first, a rotary knife sections the embedded gland starting at its square face. (To facilitate the sectioning procedure, each section is further cut in quadrants). Each section is $4 \mu m$ thick and the interval between neighboring sections is $1.5 \mathrm{~mm}$. Then, each histological section is $\mathrm{H} / \mathrm{E}$ stained and microscopically examined by pathologists to label cancer ground truth. After that, the quadrants of each section are scanned using a whole slide scanner and carefully aligned into a 2D histological slice using Adobe Photoshop ${ }^{\circledR}$ by the pathologists. Finally, histological slices are converted into gray level images and resampled from their original resolution to match the resolution of MR images. Overall, MR and histological images for five prostate specimens are acquired. 


\subsection{Overall Energy Function}

Given histological image $H: \Omega_{H} \subset \Re^{3} \mapsto \Re$ and MR image $M: \Omega_{M} \subset \Re^{3} \mapsto \Re$ of the same prostate, the 3D non-rigid (deformable) registration algorithm in this paper seeks a transformation $h: \Omega_{H} \mapsto \Omega_{M}$ that warps every point $\mathbf{u} \in \Omega_{H}$ to its counterpart $h(\mathbf{u}) \in \Omega_{M}$, by maximizing an overall energy function

$$
\begin{aligned}
E(h)= & \alpha E_{\text {LandmarkSimilarity }}\left(h ;\left\{B^{H}, B^{M}\right\},\left\{I^{H}, I^{M}\right\}\right) \\
& +\beta E_{\text {CancerRegionOverlap }}\left(h ; C^{H}, C^{M}\right) \\
& -\gamma R(h) .
\end{aligned}
$$

Here $E_{\text {LandmarkSimilarity }}$, the first registration criterion, is the similarity metric on both boundary landmarks (sets $B^{H} \& B^{M}$ ) and internal landmarks (sets $I^{H} \& I^{M}$ ) in the two images. $E_{\text {CancerRegionOverlap, the second registra- }}$ tion criterion, is the similarity metric between the cancer ground truth $C^{H}$ in histological image and the cancer region $C^{M}$ in MR image. (Note that $C^{M}$ is largely unknown and to be estimated by the registration process). $R(h)$ is the regularization of the transformation, which is described by the "bending energy" [23] of $h$ in our algorithm, i.e., $R(h)=\iiint_{(x, y, z) \in \Omega_{M}}\left(\frac{\partial^{2} h}{\partial x^{2}}+\frac{\partial^{2} h}{\partial y^{2}}+\frac{\partial^{2} h}{\partial z^{2}}\right)^{2} d x d y d z . \alpha, \beta$ and $\lambda$ are balancing parameters. The notation of each part of the registration framework is illustrated in Fig. 2.

In the subsequent sections, Section 2.3 will elaborate the 3D histology reconstruction in the pre-processing stage; based on that, Section 2.4 and 2.5 will elaborate each of the two registration criteria in details.

\subsection{Coarse Reconstruction of Histological Volume}

In the pre-processing, series of 2D histological slices are coarsely reconstructed into a $3 \mathrm{D}$ volumetric image, in order to partially account for the loss of $3 \mathrm{D}$ integrity during the histological sectioning. The construction is coarse at this pre-processing stage because it will be refined by the $3 \mathrm{D}$ non-rigid registration with MRI in the subsequent Sections 2.4 and 2.5. The coarse reconstruction is iteratively obtained; in each iteration, every histological slice is rigidly registered to the central histological slice based on mutual

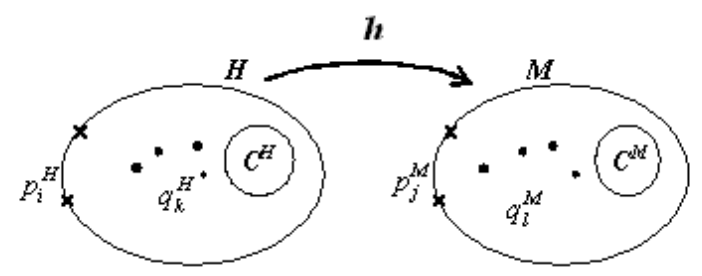

Figure 2. Notations: the registration seeks matching boundary landmarks $\left(\left\{\mathbf{p}_{i}^{H}\right\} \&\left\{\mathbf{p}_{j}^{M}\right\}\right)$, internal landmarks $\left(\left\{\mathbf{q}_{k}^{H}\right\} \&\left\{\mathbf{q}_{l}^{M}\right\}\right)$, and cancer regions $\left(C^{H} \& C^{M}\right)$ between the two images. information metric, then the tentatively reconstructed 3D histological volume is affinely registered with the 3D MRI volume based on correlation coefficient metric. The choice of rigid model for across-histological-slice registration and the choice of affine model for across-volume registration agree with existing work $[5,6]$. The resultant 3D histological volume provides initialization for the subsequent $3 \mathrm{D}$ non-rigid registration with MR image.

\subsection{Registration Criterion 1: Maximization of Landmark Similarities}

The first registration criterion seeks to maximize similarities on boundary and internal landmarks, so as to capture distortions on boundary and internal blob-like structures.

Boundary Landmarks. Boundary landmarks are automatically detected as vertices on the 3D surface of the prostate capsule. They are denoted as $B^{H}=\left\{\mathbf{p}_{i}^{H} \mid i=\right.$ $1,2, \ldots, I\}$ and $B^{M}=\left\{\mathbf{p}_{j}^{M} \mid j=1,2, \ldots, J\right\}$ in histological and MR images, respectively. Each boundary landmark is represented by a feature vector $f(\cdot)$, which represents the curvature-based geometry around the landmark at various scales [15]. Similarity on two boundary landmarks $\mathbf{p}_{i}^{H} \in B^{H}$ and $\mathbf{p}_{j}^{M} \in B^{M}$ is measured by the Euclidean distance between their geometric feature vectors - smaller distance indicates higher similarity between them,

$$
S I M_{\mathrm{Bnd}}\left(h\left(\mathbf{p}_{i}^{H}\right), \mathbf{p}_{j}^{M}\right)=-\left\|f\left(h\left(\mathbf{p}_{i}^{H}\right)\right)-f\left(\mathbf{p}_{j}^{M}\right)\right\| .
$$

Internal Landmarks. Internal landmarks are automatically detected as centers of blob-like structures using a scale space analysis method [16]. They are denoted as $I^{H}=$ $\left\{\mathbf{q}_{k}^{H} \mid k=1,2, \ldots, K\right\}$ and $I^{M}=\left\{\mathbf{q}_{l}^{M} \mid l=1,2, \ldots, L\right\}$ in histological and MR images, respectively. Similarity on two internal landmarks $\mathbf{q}_{k}^{H} \in I^{H}$ and $\mathbf{q}_{l}^{M} \in I^{M}$ is defined as the normalized mutual information (NMI)[17] between the two blobs $N_{H}\left(\mathbf{q}_{k}^{H}\right)$ and $N_{M}\left(\mathbf{q}_{l}^{M}\right)$, i.e.,

$$
S I M_{\text {Int }}\left(h\left(\mathbf{q}_{k}^{H}\right), \mathbf{q}_{l}^{M}\right)=N M I\left(N_{H}\left(h\left(\mathbf{q}_{k}^{H}\right)\right), N_{M}\left(\mathbf{q}_{l}^{M}\right)\right) \text {. }
$$

Total Similarities on Landmarks. The total landmark similarities (including boundary and internal) to be maximized are then defined as:

$$
\begin{aligned}
& \max _{h} E_{\text {LandmarkSimilarity }}\left(h ;\left\{B^{H}, B^{M}\right\},\left\{I^{H}, I^{M}\right\}\right) \\
& =\sum_{i=1}^{I} \sum_{j=1}^{J} a_{i j}\left[S I M_{\text {Bnd }}\left(h\left(\mathbf{p}_{i}^{H}\right), \mathbf{p}_{j}^{M}\right)\right] \\
& +\sum_{k=1}^{K} \sum_{l=1}^{L} \frac{1}{2} b_{k l}\left[S I M_{\text {Int }}\left(h\left(\mathbf{q}_{k}^{H}\right), \mathbf{q}_{l}^{M}\right)+S I M_{\text {Int }}\left(\mathbf{q}_{k}^{H}, h^{-1}\left(\mathbf{q}_{l}^{M}\right)\right)\right] \\
& +\left[\sum_{i=1}^{I} \sum_{j=1}^{J} a_{i j} \log \left(a_{i j}\right)+\sum_{k=1}^{K} \sum_{l=1}^{L} b_{k l} \log \left(b_{k l}\right)\right]
\end{aligned}
$$


Here $a_{i j} \in[0,1]\left(b_{k l} \in[0,1]\right)$ is the fuzziness of the matching between two boundary (internal) landmarks $\mathbf{p}_{i}^{H}$ and $\mathbf{p}_{j}^{M}\left(\mathbf{q}_{k}^{H}\right.$ and $\left.\mathbf{q}_{l}^{M}\right)$. They are defined the same as those in $[18,13,14,19,20]$, satisfying $\sum_{j=1}^{J+1} a_{i j}=1$ and $\sum_{l=1}^{L+1} b_{k l}=1$, with an extra column $\left((J+1)^{t h}\right.$ and $\left.(L+1)^{t h}\right)$ to handle outliers. Transformation is modeled by thin-plate-spline (TPS) [23], a commonly-used model that minimizes the "bending energy" of the transformation $h$.

Remarks. The similarity definitions and landmark matching processes in Eqns. (2)(3)(4) have the following three merits to promote matching accuracy and reliability:

1. In our algorithm, boundary landmarks are matched by the surface geometries around them, and internal landmarks are matched by the anatomicallymeaningful blob-like structures around them. The geometric/anatomic property based matching is relatively independent of the underlying intensity distributions. Therefore, although the intensity distributions from histological and MR images do not follow consistent relationships, where traditional mutual information [4] based matching methods tend to fail, our algorithm could establish reliable correspondences between those two images.

2. The fuzzy correspondence weights $\left\{a_{i j}\right\}$ and $\left\{b_{k l}\right\}$ are iteratively updated in an annealing process. In this annealing process, landmark correspondences converge from fuzzy to deterministic, transformation converges from coarse to fine, and the accuracies of both processes are improved. Due to the space limitation, readers are referred to [18] for more details.

3. Forward-backward matching mechanism is used to encourage two-way matching uniqueness. Ideally, correspondence should be established between a histological internal landmark $\mathbf{q}_{l}^{H}$ and a MR internal landmark $\mathbf{q}_{k}^{M}$ if and only if a) in the forward matching $(h), \mathbf{q}_{k}^{M}$ is most similar to $\mathbf{q}_{l}^{H}$ among all MR internal landmarks; and b) in the backward matching $\left(h^{-1}\right), \mathbf{q}_{l}^{H}$ is most similar to $\mathbf{q}_{k}^{M}$ among all histological internal landmarks. In extension of [13], which only performed matching in the forward direction, our approach enforces forward-backward matching consistence, therefore encourages two-way matching uniqueness.

\subsection{Registration Criterion 2: Maximization of Cancer Region Overlap}

Those boundary and internal landmarks described in the first registration criterion aim to capture 2D/3D distortions at prostate boundary and internal blob-like structures. However, due to the lack of blob-like structures around cancer regions, very few or even no internal landmarks can be detected within and around cancer regions. Consequently, the distortions around cancer regions are less likely to be captured. This causes a severe problem, since cancer regions are the main regions of interest in our histology-MRI registration context (recall that our goal is to use the registration to warp cancer regions from histology to MRI).

To specifically capture distortions at cancer regions, the second registration criterion is proposed: maximization of cancer region overlap between the two images, i.e.,

$\max _{h ; C^{M}} E_{\text {CancerRegionOverlap }}\left(h ; C^{H}, C^{M}\right)=\frac{\left|h\left(C^{H}\right) \cap C^{M}\right|}{\left|h\left(C^{H}\right) \cup C^{M}\right|}$,

where $|\cdot|$ is the cardinality of a set, $C^{H}$ is the pathologistdefined cancer ground truth region in histological image, and $C^{M}$ is the actual cancer region in MR image, which is to be segmented in the MR image but the segmentation itself is a challenging task that will be addressed below.

Rationale for Joint Cancer Segmentation and Registration. Note, however, that there are two unknowns in Eqn. (5): $h$, the registration between two images, and $C^{M}$, the cancer segmentation in MR image. Those two unknowns represent two interleaved processes that can potentially benefit each other - better registration $h$ can provide a better initialization $h\left(C^{H}\right)$ for more accurately segmenting $C^{M}$ in MR image; in return, a more accurately segmented MR cancer region $C^{M}$ could provide additional correspondences with the histological cancer regions $C^{H}$, leading to better registration $h$ between the two images.

To take advantage of the mutual benefits between those two interleaved processes, we herein propose a joint cancer segmentation and image registration framework. This framework is initialized by the registration obtained from Eqn. (4); after initialization, cancer segmentation and registration refinement are iteratively conducted. Those two processes are described below - given the tentative registration $h_{z-1}$ from the $(z-1)^{t h}$ iteration, arriving at better cancer segmentation $C_{z}^{M}$ and thereafter the registration refinement $h_{z}$ in the $z^{\text {th }}$ iteration.

Cancer Segmentation. Cancer segmentation is obtained based on learning cancer characteristics in MR image from the tentatively warped cancer regions (e.g., the regions outlined by the red contour in Fig. 3(a)). As can be observed from Fig. 3(a), the learning-based segmentation in MR image encounters two difficulties: 1) MRI intensities are often inhomogeneous and subtle-varying, causing voxels of the same tissue type to appear differently; and 2) cancer regions in MRI usually do not have clear edges/boundaries, so that the traditional edge-driven methods tend to fail.

To address those two difficulties, our segmentation consists of two steps, as shown in Fig. 3: 1) generating a cancer probability map in MR space (c.f. Fig. 3(b)) - in this way, the inhomogeneous and subtle-varying MR image intensities are converted into homogenous cancer probabilities 


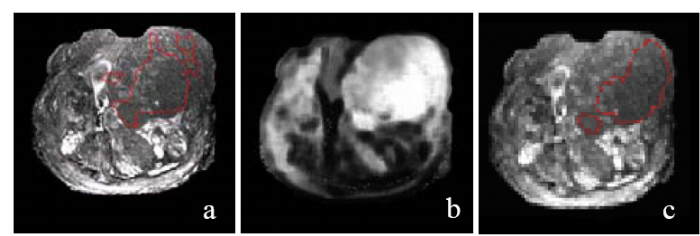

Figure 3. Demonstration of the two steps in the learning-based, region-driven cancer segmentation. (a) MRI image, with the tentatively warped cancer region outlined by red contour; (b) Probability map, generated by learning cancerous tissue characteristics from the warped cancer regions; (c) Segmented cancer region (in red contour), by evolving a deformable model that is region-driven rather than edge/boundary-driven.

[24]; and 2) segmenting cancer regions by evolving a deformable model on the probability map (c.f. Fig. 3(c)) - the segmentation is region-driven rather than edge/boundarydriven, in order to produce reliable results even when cancer boundary/edge can not be clearly detected [27, 26].

Cancer probability map is generated by a supervised classifier (support vector machine (SVM) [25] in this study). The classifier learns Gray Level Co-occurrence Matrix (GLCM) [22] textures of cancerous voxels in the tentatively warped region $h_{z-1}\left(C^{H}\right)$ and subsequently assigns a cancer probability to each voxel in the MR image. The resulting cancer probability map is denoted as $\operatorname{Pr}(\cdot)$. SVM classifier is chosen because it incorporates an implicit sample selection mechanism [25], which is capable of removing outliers that have been incorrectly included into the warped cancer regions because of registration errors at this stage.

On the probability map $\operatorname{Pr}(\cdot)$, an evolving surface $\Gamma$ is used to refine cancer segmentation in this $z^{\text {th }}$ iteration, leading to $C_{z}^{M}$ in the MR image. The evolving surface $\Gamma$ is initialized with the surface constructed from the warped cancer region $h_{z-1}\left(C^{H}\right)$. It then evolves to segment cancer region $C_{z}^{M}$, by maximizing the following energy function $\varepsilon(\Gamma)$ in a level-set implementation, i.e.,

$$
C_{z}^{M}=\operatorname{inside}\left(\Gamma^{*}\right),
$$

$$
\begin{aligned}
& \text { where } \\
& \text { and } \varepsilon(\Gamma)=\lambda_{1} \underbrace{\Gamma_{\mathbf{x} \in \text { inside }(\Gamma) \subset \Re^{3}} \operatorname{Pr}(\mathbf{x}) d \mathbf{x}}_{\text {Overall Cancer Likelihood }} \\
& -\lambda_{2} \underbrace{\lambda_{\mathbf{x} \in \text { inside }(\Gamma) \subset \Re^{3}}\left|\operatorname{Pr}(\mathbf{x})-\bar{m}_{\text {In }}\right| d \mathbf{x}}_{\text {Cancer Region Inhomogeneity }} .
\end{aligned}
$$

Here $\bar{m}_{\mathrm{In}}=\frac{\int_{\mathbf{x} \in \text { inside }(\Gamma) \subset \Re^{3}} \operatorname{Pr}(\mathbf{x}) d \mathbf{x}}{\int_{\mathbf{x} \in \text { inside }(\Gamma) \subset \Re^{3}} d \mathbf{x}}$ is the mean cancer probability inside the evolving surface. $\lambda_{1}$ and $\lambda_{2}$ are the empirically determined balancing parameters.
Understanding the two terms in Eqn. (6c) is essential to the understanding of the region-driven cancer segmentation in our approach. Generally, those two terms in Eqn. (6c) aim to locate the evolving surface $\Gamma$ at places such that 1 ) voxels inside the surface are overall most likely to be cancer (the first term); and 2) voxels inside the surface are most similar to each other so they all belong to the same tissue type, and the segmented region is therefore most homogeneous (the second term). Accordingly, the first term tends to expand the surface, because the overall cancer likelihood inside the surface will increase if the surface includes more voxels; whereas the second term tends to shrink the surface, because the regional inhomogeneity (also the voxelwise variation) will decrease if the surface includes less voxels. Both terms rely on regional information other than edge/boundary information, therefore the segmentation is purely region-driven, and is capable to arrive at reliable segmentation results, even though the cancer boundary is difficult to be detected directly. Meanwhile, the implementation is based on level set formulation, so that it can accommodate to the topology variations of cancer regions.

Refinement of Registration. The tentative cancer segmentation $C_{z}^{M}$ in MRI in the $z^{\text {th }}$ iteration provides additional correspondences with cancer regions $C^{H}$ in the histological image. This additional correspondence help refine registration from $h_{z-1}$ to $h_{z}$, such that the cancer region overlap is maximized in this $z^{t h}$ iteration, i.e.,

$$
h_{z}=\arg \max _{h} E_{\text {CancerRegionOverlap }}\left(h ; C^{H}, C_{z}^{M}\right) .
$$

Here $E_{\text {CancerRegionOverlap }}(\cdot ; \cdot, \cdot)$ is defined in Eqn. (5). $C_{z}^{M}=\operatorname{inside}\left(\Gamma^{*}\right)$ is the cancer segmentation result by the evolving surface in the $z^{\text {th }}$ iteration. In implementation of Eqn. (7), the refined registration $h_{z}$ is obtained by matching surfaces between the segmented cancer region $C_{z}^{M}$ in MR image and the ground truth cancer region $C^{H}$ in histological image, using an adaptive surface matching method in [15].

The arrival at $h_{z}$ then finishes the $z^{\text {th }}$ loop of cancer segmentation and registration refinement. This loop iterates until convergence. Convergence is satisfied when the cancer region overlap between two successive iterations reaches a high percentage such as $95 \%$.

It is worth noting that the idea of interleaving segmentation and registration in a unified framework was first developed perhaps in [29]. Since then it had found successful applications in a number of studies [30, 31, 32, 33, 24, 34]. A distinctive feature of our joint cancer segmentation and registration approach is that, it is specifically designed for images from two fundamentally different imaging modalities (i.e., histology and MRI), while others were for images necessarily from similar or even identical modalities (e.g., MRI). 


\subsection{Summary of the Algorithm}

Fig. 4 summarizes the whole registration algorithm. Our algorithm begins with coarsely reconstructing series of $2 \mathrm{D}$ histological slices into 3D volume. Then it pursues the registration in $3 \mathrm{D}$, first by maximizing boundary and internal landmark similarities (the first registration criterion). After that, the algorithm deals specifically with the registration of cancer regions - the regions of interest in our study - by maximizing cancer region overlap (the second registration criterion), which is implemented in a joint cancer registration and image registration framework. Those two criteria together lead to the final 3D non-rigid registration between histological and MR images (overall energy function).

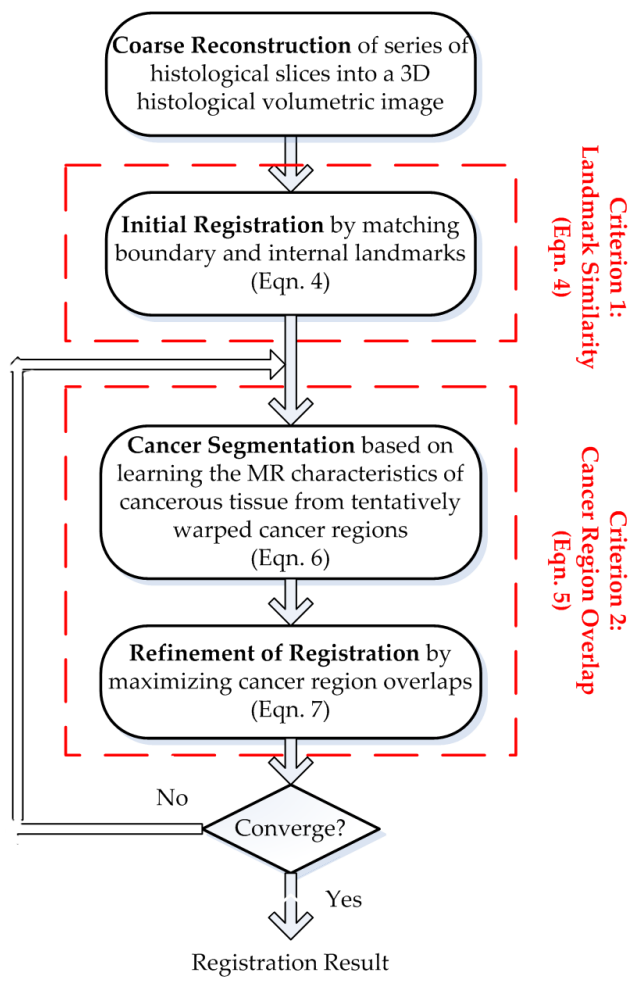

Figure 4. Summary of our non-rigid registration algorithm.

\section{Results}

Our algorithm is validated on the histological and MR image pairs described in Section 2.1. All experiments were operated in C code on a $2.8 \mathrm{G}$ Intel Xeon processor with UNIX operation system. The computational time for registering two images of size $256 \times 256 \times 64$ is typically around 25 minutes. This includes a) the 3D coarse reconstruction of histology in the pre-processing stage, b) the maximization of landmark similarities (criterion 1), and c) the maximization of cancer region overlap (criterion 2). For each of those three components, qualitative and/or quantitative results on simulated and/or real data are provided. Finally,

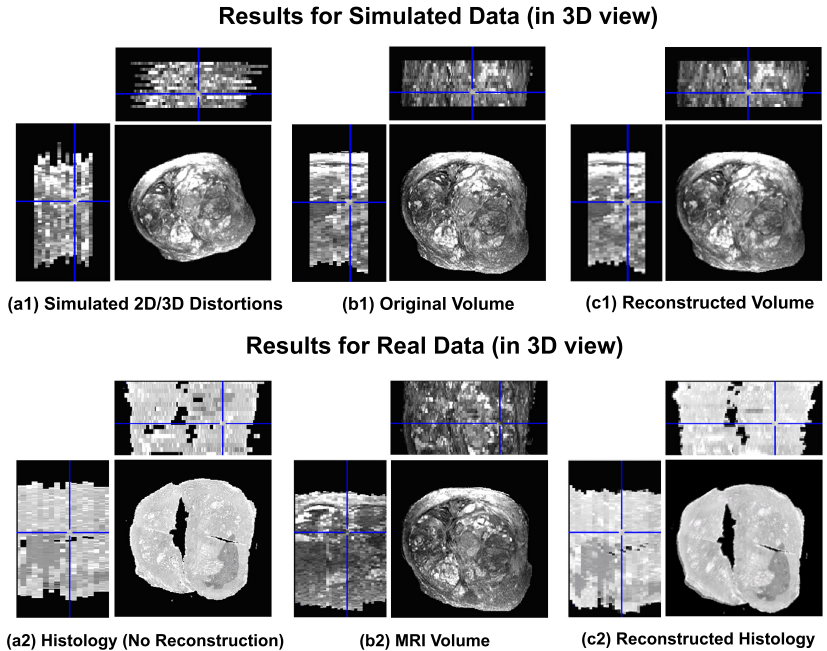

Figure 5. Coarse reconstruction for simulated (top row) and real (bottom row) data. (a1,a2) Series of distorted slices stacked together without reconstruction; (b1,b2) Reference volume; (c1,c2) Reconstructed volume. In each sub-figure, bottom left - sagittal view; bottom right - axial view; top right: coronal view.

the overall registration accuracy is established by comparing against human-rater-defined landmarks and by comparing with other registration methods.

\subsection{Results for Coarse Reconstruction of Histology}

As shown in Fig. 5, experiments on simulated and real data are provided to show that the coarse reconstruction is able to capture the linear part of the $3 \mathrm{D} / 2 \mathrm{D}$ distortions and to partially recover the 3D integrity of the volumetric image. In the simulated case, we have simulated 2D/3D linear distortions, by first applying 3D affine distortion on the original volume, followed by series of $2 \mathrm{D}$ distortions (different slices undergo different 2D distortions independently), resulting in the series of 2D/3D distorted slices in Fig. 5(a1). The reconstructed volume in Fig. 5(c1) has recovered the linear 2D/3D distortions almost perfectly. Then, the same reconstruction method is applied to reconstruct the series of real histological slices (Fig. 5(a2)), with results in Fig. 5(c2). This provides a good initialization for the subsequent 3D non-rigid registration with MRI volume.

Note that this coarse reconstruction only captures the linear part of the 2D/3D distortions and only partially recovers the $3 \mathrm{D}$ integrity at this stage; the non-linear distortions are left for the subsequent 3D non-rigid registration process.

\subsection{Results for Max. Landmark Similarities}

Fig. 6 shows results for the detection and matching of the boundary and internal landmarks. They are conducted in 3D, but for display purpose, the matched landmarks are only shown in 2D in Fig. 6. 


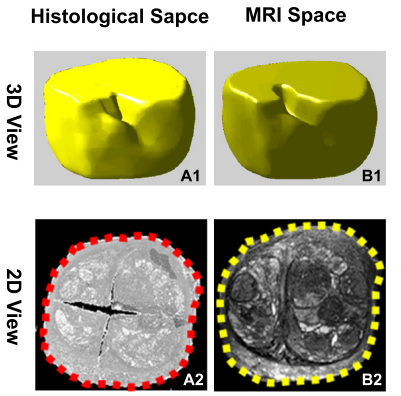

(a) Boundary Landmark Matching

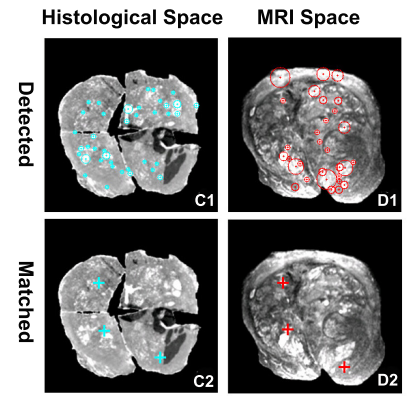

(b) Internal Landmark Matching

Figure 6. Boundary and internal landmark detection and matching results. (A1,B1) Surface of prostate capsule. (A2,B2) Corresponding boundary landmarks (red and yellow dots). (C1,D1) Detected internal landmarks (not matched) by blue and red circles; (C2,D2) Corresponding internal landmarks by blue and red crosses.

\subsection{Results for Max. Cancer Region Overlap}

The second registration criterion aims to increase registration accuracy specifically for the cancer regions, and is satisfied by jointly solving two interleaved problems - cancer segmentation and registration (Section 2.5).

Fig. 7 demonstrates how those two interleaved processes benefit each other. On one hand, the tentative registration warps the histologically-defined cancer ground truth (red contour in Fig. 7(A)) onto MR image. The warped region (yellow contour in Fig. 7(B)(C)) provides prior knowledge of cancer characteristics in MRI. Based on this prior knowledge, cancer regions can be more accurately segmented, as the segmented result (blue contour in Fig. 7(C)(D)) is now closer to the manually-delineated cancer regions (red contour in Fig. 7(D)). In this way, registration benefits cancer segmentation by providing prior knowledge of cancer characteristics. On the other hand, the segmented cancer region in MR image (blue contour in Fig. 7(C)(D)) provides additional correspondence with its counterpart in histological image (red contour in Fig. 7(A)), which can be used to improve registration accuracy. In this way, cancer segmentation also benefits registration. Overall, it is their mutual benefits that motivate the joint cancer segmentation and registration framework.
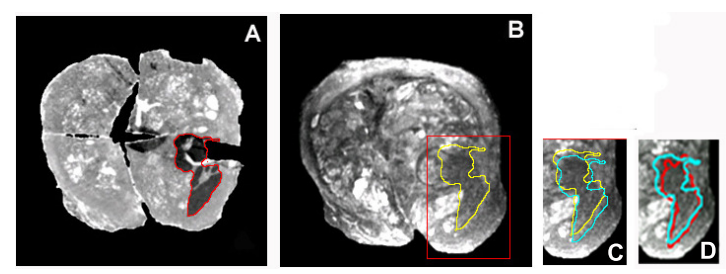

Figure 7. Demonstration of the mutual benefits between cancer segmentation and registration, and their roles in promoting registration accuracy at cancer regions. Please refer to text for details.

\subsection{Overall Accuracy}

Registration accuracy is established by a) comparing against landmarks defined by two independent human raters, and b) comparing with other registration methods. For the former, two raters independently defined correspondences on anatomically salient landmarks. The landmark errors between registered results and manual definitions are listed in Table 1, which shows that the accuracy of our algorithm is comparable to that of the human experts' visual registration. Results for the latter is shown in Table 2, where the registration accuracies of four different methods (including ours) are compared, in terms of the overlap between the warped cancer regions in MR image and the manually label cancer regions in MR image - higher overlap indicates higher accuracy. From Table 2, our method has obtained the highest registration accuracy. The significant improvement of accuracy over the third method (method M3 [13], based on boundary and internal landmarks) underlines the advantage of introducing the additional registration criterion specifically at cancer regions.

Table 1. Comparison of landmark errors among human raters and our algorithm (R1-rater1; R2-rater2).

\begin{tabular}{c|c|c|c}
\hline \hline Diff & R1 vs R2 & R1 vs Ours & R2 vs Ours \\
\hline Mean $(\mathrm{mm})$ & 0.93 & 0.62 & 0.96 \\
Std $(\mathrm{mm})$ & 0.65 & 0.43 & 0.79 \\
\hline
\end{tabular}

Table 2. Overlap between warped and manually labeled cancer region in MRI, by different methods. (M1-mutual information (MI)based affine method [4]; M2-surface matching method [15]; M3boundary and internal landmark based method [13].)

\begin{tabular}{c|c|c|c|c}
\hline \hline Overlap & M1 & M2 & M3 & Ours \\
\hline Max & $82.9 \%$ & $87.5 \%$ & $88.3 \%$ & $\mathbf{9 5 . 4 \%}$ \\
Min & $55.9 \%$ & $60.4 \%$ & $64.1 \%$ & $\mathbf{7 9 . 6 \%}$ \\
Mean & $71.6 \%$ & $75.5 \%$ & $79.1 \%$ & $\mathbf{8 5 . 4 \%}$ \\
\hline
\end{tabular}

\section{Discussion}

This paper presents a 3D non-rigid registration algorithm between histological and MR images of the same prostate. To compensate for the loss of 3D integrity during histology sectioning, our algorithm initializes with the coarse reconstruction of series of 2D histological slices/sections into a $3 \mathrm{D}$ volume. Then, to cope with the distortions in histological images and the fundamental imaging differences between histology and MRI, our algorithm registers the two images by maximizing landmark similarities and cancer region overlap between the two images. The former aims to capture distortions at prostate boundary and internal blob-like structures; and the latter aims to capture distortions specifically at the cancer regions. The overall registration accuracy is established by comparing against humanrater-defined landmarks and by comparing with other methods. With this registration, the histologically-defined cancer 
ground truth can be warped to MR images, promoting more thorough understanding of the MR characteristics of the prostate cancerous tissue, which will help the MRI-based prostate cancer diagnosis in the future studies.

The main contributions of this work are the introduction and the implementation of the second registration criterion - maximization of cancer regions. By introducing this criterion, registration accuracy within and around cancer regions has been significantly improved, as qualitatively shown in Fig. 7 and quantitatively demonstrated in Table 2 . This is important because cancer regions are the regions of interest in this histology-MRI registration context (keep in mind that our objective is to warp cancer regions from histology to MRI). In implementing this criterion, a joint cancer segmentation and registration framework is proposed, where the two interleaved processes benefit each other and iteratively increase the overall accuracy. Furthermore, the cancer segmentation is region-driven other than edge-driven, which is more reliable when the cancer edges/boundaries are difficult to be detected in MR images.

Our future work calls for a more sophisticated transformation mechanism to better deal with the histological cuts/tears (c.f., Fig. 1), and the consequent loss of correspondence. Actually, lack of such a sophisticated mechanism is an inherent limitation for most histology-MRI registration methods including ours. Our plan is to develop a well-formulated "confidence"-based mechanism. In this mechanism, those regions having high confidence to establish correspondences will become the main driving force for deformations, and those regions having difficulty establishing correspondences (such as histological cuts/tears) will have low impact for the deformation. A recently developed method [28] uses this confidence mechanism and shows promise to reduce the negative impact of cuts/tears in the simulated data. More experiments are still expected on real histology-MRI data.

In conclusion, this paper presents a 3D non-rigid registration algorithm between histological and MR images of the same prostate. Future work calls for a more sophisticated transformation mechanism to better deal with histological cuts/tears, and more validations on real data.

\section{References}

[1] Hricak, H., et al., "Imaging prostate cancer: a multidisciplinary perspective". Radiology, 243(1): p. 28-53, (2007).

[2] Kirkham, A., et al., "How good is MRI at detecting and characterizing cancer within the prostate?" Eur Urol. 50: 1163-1174, (2006).

[3] Cancer Facts and Figures, 2008. American Cancer Society.

[4] W. Wells, III, et al, "Multi-modal volume registration by maximization of mutual information," MedIA. 1: 35-51, (1996).

[5] Ourselin, S., et al, "Fusion of Histological Sections and MR Images: Towards the Construction of an Atlas of the Human Basal Ganglia", MICCAI: 743-751, (2001).

[6] Bardinet, E., et al, "Co-registration of Histological, Optical and MR Data of the Human Brain", MICCAI: 548-555, (2002).

[7] Pitiot, A., et al, "Piecewise affine registration of biological images for volume reconstruction", Med. Im. Ana., 10(3): 465-483, (2006).
[8] Jacobs, M.A., et al: "Registration and warping of magnetic resonance images to histological sections". Med. Phy. 26: 1568-1578. (1999)

[9] Li, G., et al: 'Registration of in vivo magnetic resonance T1-weighted brain images to triphenyltetrazolium chloride stained sections in small animals". J. Neur. Methods. 156(1-2): 368-375. (2006)

[10] Breen, M., et al: "Correcting spatial distortion in histological images". Comp. Med. Imag. Grap. 29(6): 405-417. (2005)

[11] Meyer, CR, et al: "A methodology for registration of a histological slide and in vivo MRI volume based on optimizing mutual information". Mol. Imaging. 5(1): 16-23. (2006)

[12] Dauguet, J., et al: "Three-dimensional reconstruction of stained histological slices and 3D non-linear registration with in-vivo MRI for whole baboon brain”. J. of Neur. Methods. 164: 191-204. (2007)

[13] Zhan, Y., et al: "Registering Histologic and MR Images of Prostate for Image-based Cancer Detection". Academic Radiology. 14(11): 1367-1381. (2007)

[14] Davatzikos, C., et al: "Correspondence detection in diffusion tensor images". ISBI: 646-649 (2006)

[15] Shen, D., et al: "An adaptive focus statistical shape model for segmentation and shape modeling of 3D brain structures". IEEE TMI., 20: 257-271. (2001)

[16] Lindeberg T.: "Feature detection with automatic scale selection". Intl. J. of Comp. Vision, 30: 77-116. (1998)

[17] Maes, F., et al: 'Multimodality Image Registration by Maximization of Mutual Information". IEEE TMI. 16: 187-198. (1997)

[18] Chui, H. and Rangarajan, A.: "A new point matching algorithm for non-rigid registration". Computer Vision and Image Understanding. 89:114-141. (2003)

[19] Yang, J., et al, "Non-rigid Image registration using geometric features and local salient region features". CVPR, 2006.

[20] Shen, D., "Fast image registration by hierarchical soft correspondence detection", Pattern Recognition, 42(5): 954-961, (2009).

[21] Christensen, G.E and Johnson, H.J.: "Consistent Image Registration". IEEE Trans. Med. Imag.. 20(7): 586-582. (2001)

[22] Haralick, R.M.: "Statistical and structural approaches to texture". Proceedings of the IEEE, 67(5): 786-806. (1979)

[23] Bookstein, F.L: "Principal Warps: Thin-Plate Splines and the Decomposition of Deformations". IEEE Transactions on PAMI. 11(6): 567-585. (1989)

[24] Xing, Y., et al, 'Improving Parenchyma Segmentation by Simultaneous Estimation of Tissue Property T1 Map and Group-Wise Registration of Inversion Recovery MR Breast Images”. MICCAI: 342-350, (2008).

[25] Burges, C., "A tutorial on support vector machines for pattern recognition". Data Mining and Knowledge Discovery, 2(2): p. 121-167, (1998).

[26] Chan, T.F. and Vese, L.A.: "Active Contour Without Edges". IEEE Trans. Image Processing. 10(2): 266-277. (2001)

[27] Mumford, D., Shah, J.: "Optimal Approximation by Piecewise Smooth Functions and Associated Variational Problems". Comm. Pure and Applied Math. 42: 577-685. (1989)

[28] Ou, Y. and Davatzikos, C. "DRAMMS: Deformable Registration via Attribute Matching and Mutual-Saliency weighting", Info. Proc. in Med. Imag. (IPMI), 2009.

[29] Yezzi, A., et al. "A variational framework for joint segmentation and registration". in MMBIA, 2001.

[30] Wyatt, P. and Noble J.A., "MAP MRF joint segmentation and registration of medical images", Med. Im. Ana., 7, 539-552, (2003).

[31] Chen, X., et al. "Simultaneous segmentation and registration of contrast-enhanced breast MRI". IPMI: 126-137, (2005).

[32] Pohl, K., et al. "A Bayesian model for joint segmentation and registration". Neuroimage, 31: 228-239, (2006).

[33] Wang, F., et al. "Joint registration and segmentation from brain MRI", Acad Rad, 13(9): 1104-11. (2006).

[34] Xue, Z., Wong, K., Wong, S.: "Joint Registration and Segmentation of Serial Lung CT Images in Microendoscopy Molecular ImageGuided Therapy". MIAR: 12-20, (2008). 